\title{
Addressing the Terrain Topography in Distributed SAR Imaging
}

\author{
Thomas Kraus, Gerhard Krieger, Markus Bachmann, Alberto Moreira \\ Microwaves and Radar Institute \\ German Aerospace Center (DLR) \\ Oberpfaffenhofen, Germany \\ t.kraus@dlr.de
}

\begin{abstract}
Distributed multichannel synthetic aperture radar (SAR) imaging is a promising concept for future Earth observation missions. The multichannel concept can mitigate the pulse repetition frequency (PRF) or minimum SAR antenna area constraints inherent to single-channel SAR systems. Thus high azimuth resolution can be maintained, while acquiring wide swathes. This enables global coverage Earth observation with high spatial and temporal resolution. An important step during the multichannel processing for distributed SAR systems is the compensation of the topographic phase. This phase is the result of non-zero cross-track baselines between the antenna phase centers in a constellation or swarm of satellites. The paper in hand investigates different approaches to consider the topography during SAR processing.

Index Terms-multistatic SAR, multichannel SAR, topography consideration
\end{abstract}

\section{INTRODUCTION}

The demand for high-resolution wide-swath (HRWS) SAR imaging is continuously growing as the capabilities to exploit SAR imagery for operational applications are developing. An example demanding wide coverage and high resolution at the same time is ship or oil spill detection over wide areas combined with ship imaging for identification. To solve the contradicting PRF requirements imposed by the simultaneous demand for high azimuth resolution and wide coverage, innovative techniques have been suggested. The concept to employ multiple channels as proposed in [1] offers the necessary degrees of freedom. This concept is generalized in [2] for non-uniform sampling conditions and further elaborated, e.g., in [3], [4], [5]. Spaceborne demonstrations of the technique for single platform systems are reported in [6] and [4]. However, an extension to distributed satellite systems is even more promising. Such a system could additionally provide interferometric and tomographic capabilities [7], [8]. First results for distributed SAR imaging are reported in [9], [10], [11]. For SAR systems based on a swarm or constellation of satellites the presence of cross-track baselines offers new opportunities as the mentioned interferometric capabilities. For a HRWS application, however, this baseline component is complicating the azimuth signal reconstruction process. A phase difference between the channels is introduced depending on the orbit parameters and the imaged topography. Compensation methods used for a TerraSAR-X and TanDEM-X experiment are discussed in [12]. The paper in hand explains these methods in more detail, further develops the underlying ideas and shows the challenges in addressing the terrain topography in distributed SAR imaging.

The paper is structured as follows. In section II the acquisition geometry of a distributed SAR system and the sampling are discussed. Section III introduces two approaches to consider the more general acquisition geometries during SAR processing. In section III-A the bandpass decomposition approach is explained and section III-B introduces the use of beamforming methods. Properties of the methods are discussed and simulation results are shown. Section IV summarizes the paper.

\section{ACQUisition Geometry AND SAMPLing}

When multichannel SAR systems are analyzed, different parameters affect the signal reconstruction. Here we focus on the acquisition geometry and the sampling of the azimuth signal below the Nyquist rate.

\section{A. Acquisition Geometry}

For multichannel SAR systems on a single satellite, only the mechanically defined along-track separation of the phase centers together with the PRF and the platform velocity are of interest for the multichannel SAR signal reconstruction. These parameters determine the resulting image performance. For a distributed SAR system, however, the acquisition geometry is more complex. Several other factors have to be considered. An overview is given in Fig. 1. A perpendicular baseline component $b_{\perp}$ leads to a range dependent interferometric phase between the channels. This is the case, even when a flat Earth model is considered as shown at the top. The center of Fig. 1 discusses the impact of a topography variation in azimuth direction, which leads to an azimuth variant fringe pattern. Finally, a line-of-sight (LOS) baseline component $b_{\text {LOS }}$ leads to different azimuth chirp rates of the signals of different channels as shown at the bottom.

\section{B. Sampling}

In general the azimuth signal is sampled below the Nyquist rate for HRWS applications. Therefore, the azimuth bandwidth of the signal received by the azimuth antenna pattern is 

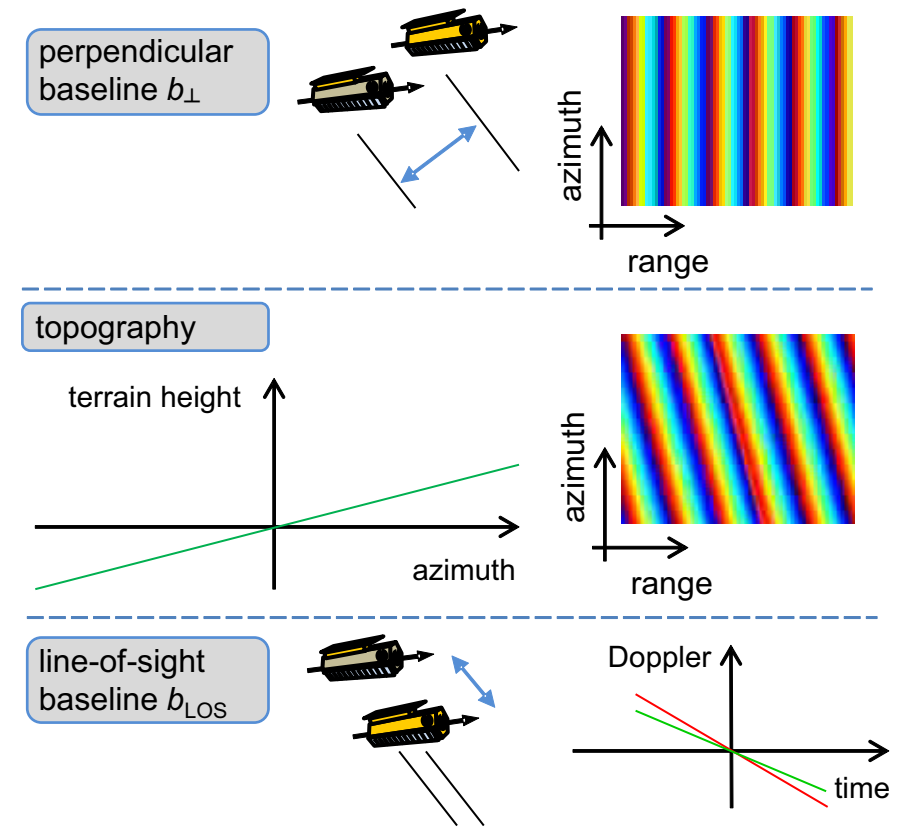

Fig. 1. Effects of the distributed SAR system acquisition geometry. Perpendicular baseline $b_{\perp}$ leads to an interferometric phase between the channels (top). Topographic variation in azimuth direction leads to an azimuth variant fringe pattern (center). A line-of-sight component of the baseline $b_{\text {LOS }}$ leads to different azimuth chirp rates (bottom).

significantly wider than the PRF band which ranges from $-f_{\mathrm{S}} / 2$ to $f_{\mathrm{S}} / 2$. This is sketched in Fig. 2 in the first and second row. The azimuth signal reconstruction approach described in [2] exploits the information contained in all channels by combining them with appropriate weights. By the weighted summation a constructive interference of the signal is achieved, whereas the ambiguous components cancel out. This is shown at the bottom of Fig. 2. The reconstructed Doppler band ranges from $-f_{\mathrm{S}}$ to $f_{\mathrm{S}}$, as indicated by the gray shaded areas. The ambiguous components of the spectra which are canceled by the reconstruction process are shown with semitransparent color.

\section{TOPOGRAPHY CONSIDERATION}

To compensate the impact of the more complex acquisition geometry of a distributed SAR system different approaches can be envisaged. One approach is to account for the effects in a dedicated processing step before the actual azimuth signal reconstruction. This step is called topography correction in [9], [11], [12]. The corrective step is necessary for all but the master channel. The goal is to adjust the slave channels in a way to end up with quasi single-platform multichannel signals. A technique following this approach is the bandpass decomposition described below. Another idea is to treat the signals in a more general way. The signals of different apertures are regarded as samples in space. By deriving appropriate weights, beamforming can be used to reconstruct and focus the azimuth signal in a single step. The well known time domain back-projection method can be regarded as one example for such a beamforming method [13], [14]. In the following

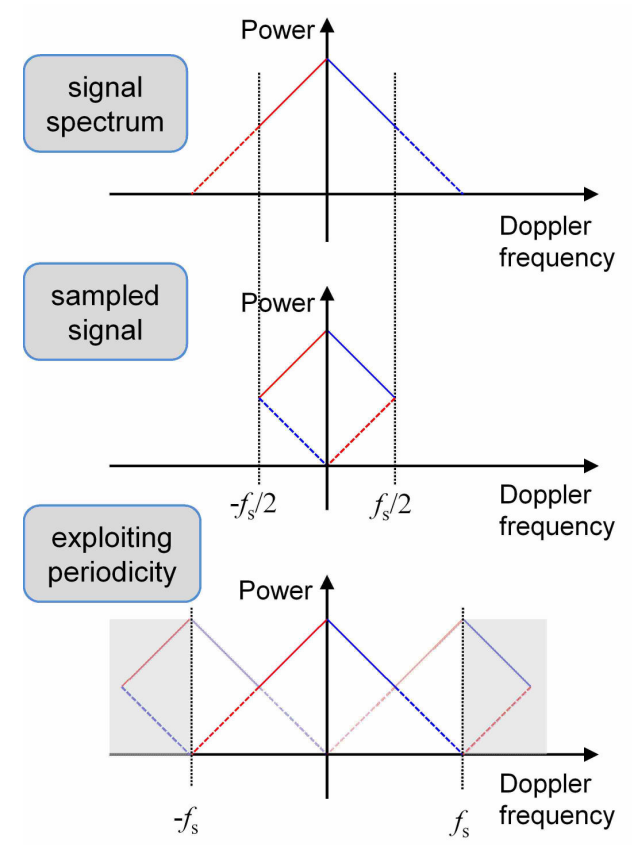

Fig. 2. Signal spectrum before (top) and after (middle) sampling by the PRF $f_{S}$. The spectrum is folded into the Doppler frequency range of one PRF. In order to identify the aliased parts of the spectrum, they are drawn in red and blue and with solid and dashed lines. By assuming a two-channel system and exploiting the spectral periodicity of the sampled signal a reconstruction filtering is possible (bottom). The aliased components, which in an ideal reconstruction are canceled out, are drawn semi-transparently. The periodic part of the spectrum beyond the reconstructed bandwidth of twice the PRF is shaded in gray.

the bandpass decomposition method and beamforming for multichannel SAR signal reconstruction are analyzed.

\section{A. Bandpass Decomposition}

The topography compensation is only necessary for the slave channels. It is performed before azimuth signal reconstruction and azimuth focusing. Since the signal is not focused in azimuth the contribution of a point like target extends over the length of the synthetic aperture, limited by the azimuth antenna pattern. The signals of other targets distributed in azimuth direction are superimposed. In general those targets are not located on the same terrain height as we consider an azimuth variant topography. The idea behind the bandpass decomposition approach is, to consider this by decomposing the azimuth signal into sub-bands. Thereby, the area seen by the azimuth antenna beam can be divided in order to resolve the topographic variation within the beam. A block diagram of the bandpass decomposition is shown in Fig. 3 . The slave channels are decomposed into bandpass signals in Doppler domain. This bandpass signals are corrected for the topographic phase in time domain based on digital elevation model (DEM) information according to the direction which corresponds to the bandpass center frequency.

Since the single channel data are aliased (cf. middle of Fig. 2) the correction of the topographic phase can only be correctly performed for one direction or Doppler frequency, 


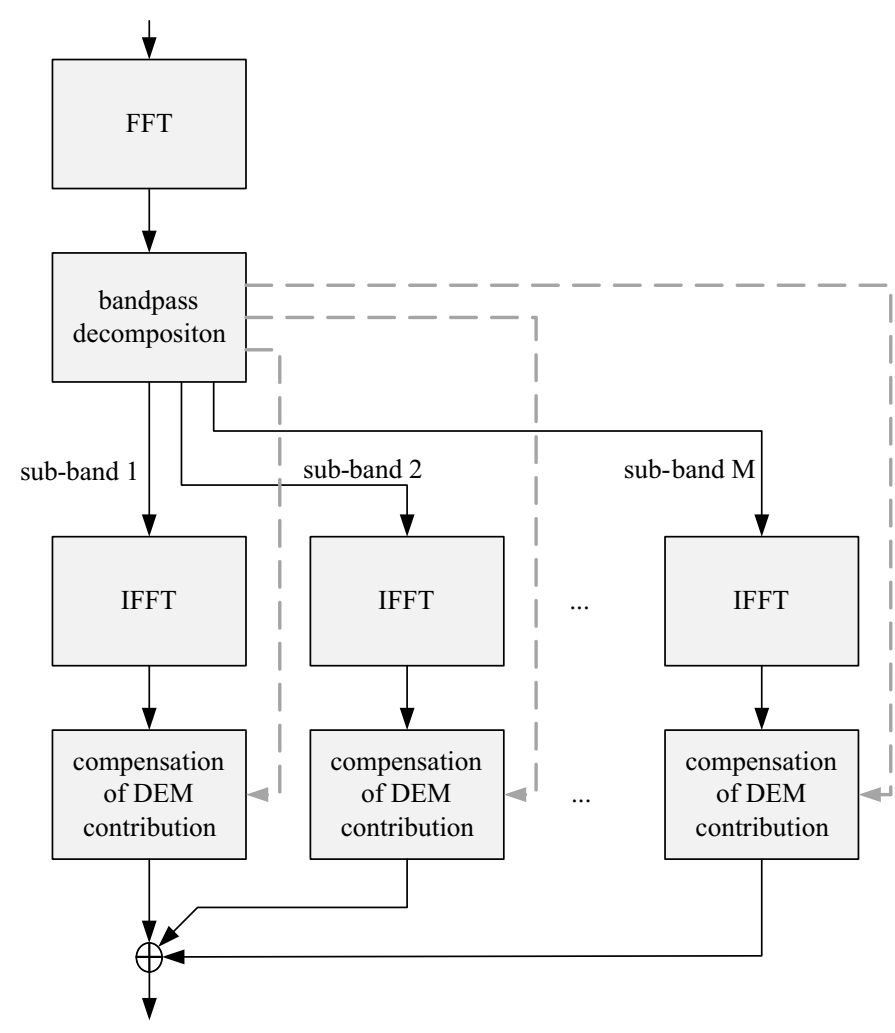

Fig. 3. Block diagram of the topography correction using the bandpass approach. The slave channels azimuth signal is transformed to the Doppler domain and decomposed into several sub-bands. The sub-band signals are transformed to time domain and corrected for the topography corresponding to the respective Doppler band. Finally the sub-band signals are summed up to get the full bandwidth topography corrected signal.

respectively. The situation is shown in Fig. 4 for a two channel system and three different possibilities. On the left side, the position of a point target is depicted by the blue circle, its ambiguities in red. The dashed red line indicates the area from which the height information is used to correct the slave channel. In the top row the terrain around the actual point target is considered, leading to constructive interference for the point targets impulse response function (IRF). However, the destructive interference of the ambiguities is not ensured. This leads to an increase of the azimuth ambiguity-to-signal ratio (AASR) for steeper slope values as depicted on the right side. Here the AASR is shown for the left and the right ambiguity of the reconstructed image separately in red and orange color. The blue line represents the single-channel AASR whereas the green line corresponds to a single-channel system with twice the PRF of the two-channel system under evaluation. In the middle row the terrain around one of the ambiguities is considered, resulting in an excellent suppression of the corresponding ambiguity. Because the other ambiguity area is not considered at all, their AASR strongly increases. As discussed in [12] this approach could be useful for ship detection near the coast, where the dominant ambiguity power originates from only one direction. The last row seems to offer a good compromise by considering the terrain in a way, resulting in symmetric and appreciably low AASR. Here the terrain is considered, from where the dominant ambiguous power is originating in order to emphasize its cancellation by the reconstruction algorithm. This approach has the disadvantage of using a step function as the terrain model. The resulting IRF suffers from strongly increased side-lobe levels and degraded resolution, disqualifying this approach for common practical applications. Nevertheless, the presented results reveal the challenges associated to distributed SAR systems to deliver high quality SAR imagery. A balanced trade has to be established between ambiguity suppression and IRF performance. This experience leads to the approach presented in the following section.
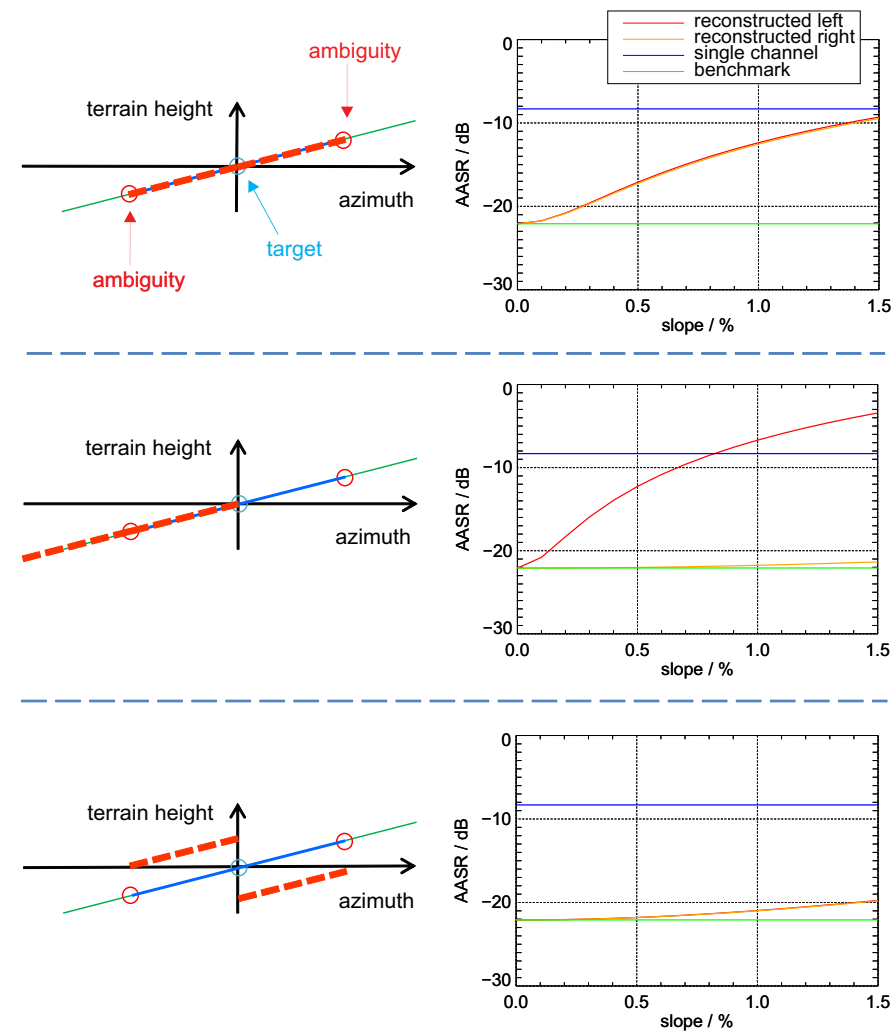

Fig. 4. Three different possibilities for which direction the topography can be corrected: Position of the actual point target (top), one of the ambiguities (middle) or for both first order ambiguities partially (bottom). The corresponding azimuth ambiguity performance versus terrain slope is depicted on the right side for an acquisition geometry with $89 \mathrm{~m}$ perpendicular baseline, simulated for a X-band system with a satellite altitude of $510 \mathrm{~km}$ and a look angle of $26^{\circ}$ in a rectilinear geometry.

\section{B. Beamforming}

Back-projection can be regarded as a simple beamforming technique. It inherently incorporates DEM information into the SAR processing chain and derives filter weight in order to maximize the IRF of a target. The position of the pixel on the DEM as well as the positions of all samples of the synthetic aperture are considered to derive the filter coefficients, as shown in Fig. 5. This technique is computationally more expensive than frequency domain algorithms, but it can handle more complex acquisition geometries. 


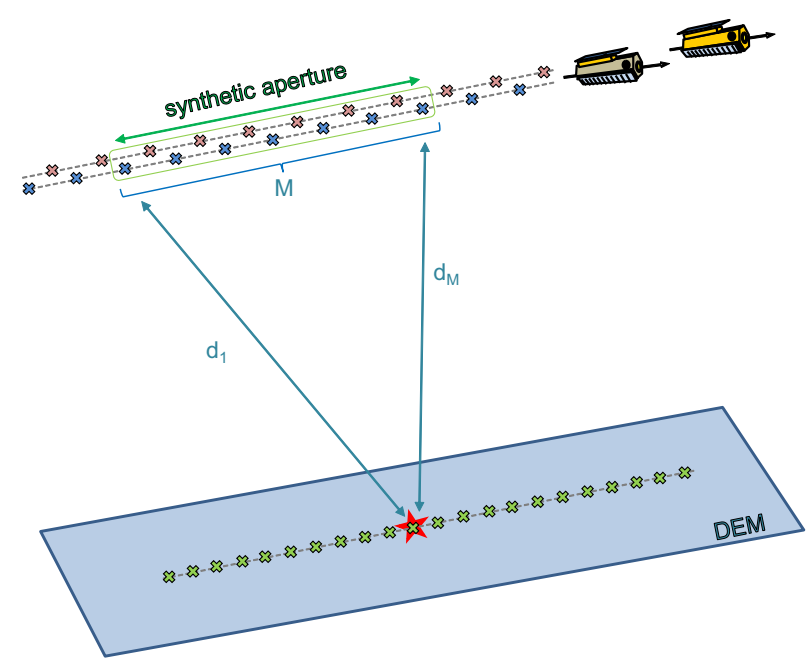

Fig. 5. Sketch of the acquisition geometry and the processing for time domain back-projection. The length of the synthetic aperture comprising $M$ samples is highlighted. The distances $d_{i}$ of the $M$ samples to the pixel which should be focused on ground are used to calculate the filter coefficients.

In Fig. 6 the AASR performance versus terrain slope is depicted resulting from back-projection processing for a satellite formation with $89 \mathrm{~m}$ perpendicular baseline and a line-of-sight baseline of $181 \mathrm{~m}$. It is simulated for an X-band system with a satellite altitude of $510 \mathrm{~km}$ and a look angle of $26^{\circ}$ in a rectilinear geometry. Compared to the results shown at the top of Fig. 4 two differences are noticeable: The ambiguity performance is not symmetric any more (for the left and the right ambiguity), even though there is no special treatment for one ambiguity. Additionally, the AASR even for slopes of $0 \%$ is not coinciding with the one of the benchmark system. The reason for both observations is the presence of the LOSbaseline. The effect can be explained by different azimuth chirp rates which are a result of slant range differences due to the baseline. Without a LOS baseline the ambiguities occur at exactly the same position for both channels at a distance to the actual target of

$$
d_{\mathrm{ambi}} \approx \frac{f_{\mathrm{S}} \lambda r_{0}}{2 v}
$$

where $r_{0}$ is the range of closest approach and $v$ is the platform velocity in a rectilinear geometry. Since the ranges of closest approach differ in the presence of a LOS baseline, the ambiguities do no longer coincide spatially and therefore cannot cancel out.

The asymmetric ambiguity behavior for non-zero slopes is also a result of the LOS baseline. In the presence of a linear terrain slope, one ambiguity appears at a position closer to the radar and one at a position further away. Without the LOS baseline, back-projection delivers the same result as depicted in Fig. 4 on the top, because in azimuth direction a uniform sampling is assumed. For a non-uniform sampling in azimuth the bandpass decomposition approach in general outperforms back-projection as bandpass decomposition builds on the reconstruction algorithm [2].

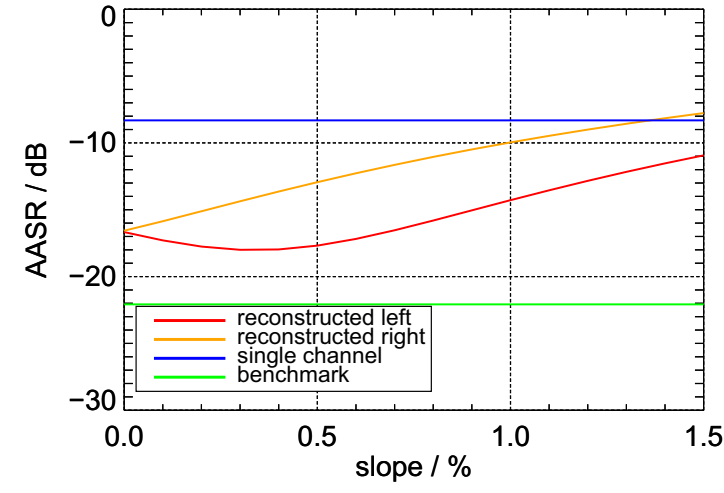

Fig. 6. AASR achieved using the back-projection approach for the correction of the topographic contribution for a perpendicular baseline of $89 \mathrm{~m}$ and a line-of-sight baseline of $181 \mathrm{~m}$. The red and orange lines represent the AASR of the combined signal. The blue one is the single-channel AASR and the green line corresponds to a monostatic system with twice the PRF of the two-channel multistatic system.

Back-projection maximizes the signal energy for a given point target on the ground. However, it does not provide any means to control the ambiguity performance. Therefore, more sophisticated beamforming techniques have to be considered [15], [16], [7]. Fig. 7 shows the geometry under consideration for the example of a three-channel system. The goal is to steer nulls to the positions of the ambiguities depicted in yellow. Another option would be to suppress the ambiguous power below a certain level, e.g., the noise level, using a sidelobe-constrained minimum variance beamformer [17]. Both techniques result in an azimuth-variant SAR focusing that resembles a large space-variant beamformer, where the beamformer weights are continuously adjusted in accordance with the satellite formation geometry and the terrain topography to maximize the energy from a given resolution cell under the constraint of minimizing the signal returns from all other directions.

For the results shown in Fig. 8, the null-steering approach is employed. For a three-channel system the results of backprojection (BP) and beamforming (BF) with nulls steered in the ambiguous directions are compared. The dashed red and orange lines correspond to back-projection, and the solid lines correspond to beamforming. The AASR achieved with the null-steering beamforming approach clearly outperforms the ambiguity performance achieved with back-projection. This is the case as the power at the dominant ambiguities positions is reduced by steering nulls in exactly these directions. Nevertheless, the beamforming results show comparable IRF characteristics as the back-projected signals. These results demonstrate the promising capabilities offered by beamforming when used for SAR processing.

\section{SUMMARY}

The paper discusses the impact of the geometry of a distributed SAR system on the azimuth signal reconstruction. Two methods are analyzed. On the one hand the bandpass decomposition approach is investigated, which aims at the 


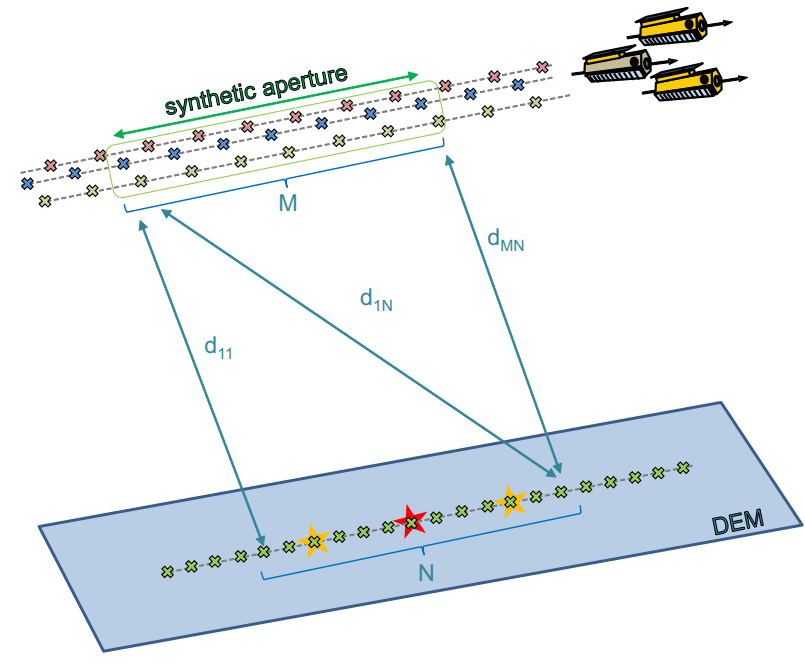

Fig. 7. Sketch of the acquisition geometry and the processing for the beamforming approach for a three-channel system. The length of the synthetic aperture comprising $M$ samples is highlighted. The distances $d_{i j}$ of the $M$ samples to $N$ pixels on ground are used to calculate the beamformer weights. The target to be focused is shown in red and its ambiguities in yellow.

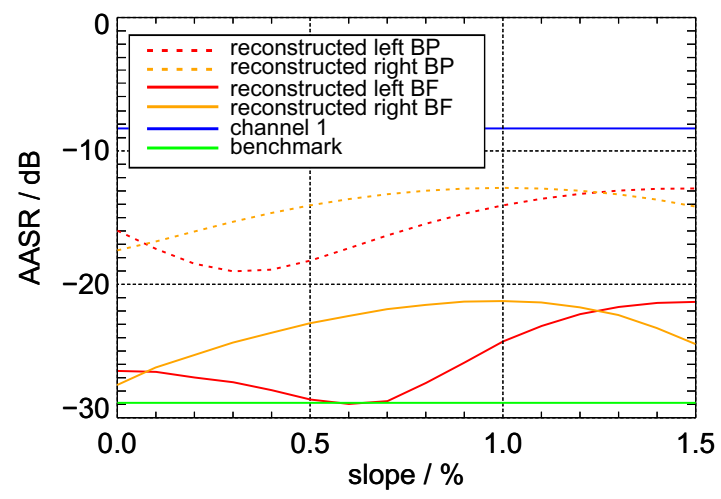

Fig. 8. AASR achieved for a three-channel system, using back-projection and the beamforming approach for the correction of the topographic contribution for a perpendicular baseline of $\pm 89 \mathrm{~m}$ and a line-of-sight baseline of \pm 181 $\mathrm{m}$. The red and orange lines represent the AASR of the combined signals. The dashed lines correspond to back-projection (BP) processing, and the solid lines correspond to beamforming (BF). The blue one is the single-channel AASR and the green line corresponds to a monostatic system with three times the PRF of the three-channel multistatic system.

preconditioning of the signals to suit the established azimuth signal reconstruction algorithm in Doppler domain. On the other hand a beamforming approach is discussed which completely works in time domain and simultaneously conducts reconstruction and SAR focusing.

The analysis of the bandpass decomposition approach delivers many interesting insights. However, its practical applicability seems to be limited. Achieving a trade off between good ambiguity suppression performance and proper IRF parameters (side-lobe levels) is difficult. The beamforming approach is promising since more degrees of freedom are available. A classical back-projection is not sufficient since it puts no emphasis on ambiguity cancellation or suppression. Using a null-steering beamforming approach delivers a superior am- biguity performance. It enables the suppression of dominant ambiguities while achieving comparable IRF characteristics as back-projection.

The presented results are promising. Nevertheless, many interesting questions remain to be analyzed. A more general geometry with non-linear satellite tracks, non-parallel orbits and slightly different platform velocities as well as nonuniformity in the azimuth sampling need to be considered. Additionally, the computational complexity and possible simplifications of the approach need to be analyzed in order to justify its practical applicability.

\section{REFERENCES}

[1] A. Currie and M. A. Brown, "Wide-swath SAR," in Proc. Inst. Elect. Eng.-Radar, Sonar, Navigat., vol. 139, no. 2, 1992, pp. 122-135.

[2] G. Krieger, N. Gebert, and A. Moreira, "Unambiguous SAR signal reconstruction from nonuniform displaced phase center sampling," IEEE Geosci. Remote Sens. Lett., vol. 1, no. 4, pp. 260-264, 2004.

[3] N. Gebert, G. Krieger, and A. Moreira, "Digital beamforming on receive: Techniques and optimization strategies for high-resolution wide-swath SAR imaging," IEEE Trans. Aerosp. Electron. Syst., vol. 45, no. 2, pp. 564-592, 2009.

[4] D. Cerutti-Maori, I. Sikaneta, J. Klare, and C. H. Gierull, "MIMO SAR processing for multichannel high-resolution wide-swath radars," IEEE Transactions on Geoscience and Remote Sensing, vol. 52, no. 8, pp. 5034-5055, 2014.

[5] I. Sikaneta, C. Gierull, and D. Cerutti-Maori, "Optimum signal processing for multichannel SAR: With application to high-resolution wideswath imaging," IEEE Trans. Geosci. Remote Sens., vol. 52, no. 10, pp. 6095-6109, 2014.

[6] J.-H. Kim, M. Younis, P. Prats-Iraola, M. Gabele, and G. Krieger, "First spaceborne demonstration of digital beamforming for azimuth ambiguity suppression," IEEE Trans. Geosci. Remote Sens., vol. 51, no. 1, pp. 579$590,2013$.

[7] G. Krieger and A. Moreira, "Potential of digital beamforming in bi-and multistatic SAR," in Geoscience and Remote Sensing Symposium, 2003. IGARSS'03. Proceedings. 2003 IEEE International, vol. 1. IEEE, 2003, pp. 527-529.

[8] — "Spaceborne bi- and multistatic SAR: potential and challenges," Proc. Inst. Elect. Eng.-Radar, Sonar, Navigat., vol. 153, no. 3, pp. 184-198, 2006.

[9] T. Kraus, B. Bräutigam, M. Bachmann, and G. Krieger, "Multistatic SAR imaging: First results of a four phase center experiment with TerraSAR-X and TanDEM-X," in Proc. EUSAR, Hamburg, Germany, 2016.

[10] T. Kraus, M. Bachmann, L. Heiderich, G. Krieger, and A. Moreira, "Multistatic SAR imaging: Comparison of simulation results and experimental data," in Proc. Inst. Elect. Eng.-Radar, Sonar, Navigat. IET, 2017.

[11] T. Kraus, G. Krieger, M. Bachmann, and A. Moreira, "Spaceborne demonstration of distributed SAR imaging with TerraSAR-X and TanDEM-X," IEEE Geosci. Remote Sens. Lett., 2019, early access.

[12] T. Kraus, M. Bachmann, and G. Krieger, "Topography correction for distributed SAR imaging: A case study for TerraSAR-X and TanDEM-X," in Proc. EUSAR, 2018

[13] L. M. Ulander, H. Hellsten, and G. Stenstrom, "Synthetic-aperture radar processing using fast factorized back-projection," IEEE Transactions on Aerospace and electronic systems, vol. 39, no. 3, pp. 760-776, 2003.

[14] M. Soumekh, Synthetic aperture radar signal processing. New York: Wiley, 1999, vol. 7.

[15] N. A. Goodman, S. C. Lin, D. Rajakrishna, and J. M. Stiles, "Processing of multiple-receiver spaceborne arrays for wide-area SAR," IEEE Trans. Geosci. Remote Sens., vol. 40, no. 4, pp. 841-852, 2002.

[16] J. M. Stiles and N. A. Goodman, "Wide area, fine resolution SAR from multi-aperture radar arrays," in Proc. of the Advanced SAR Workshop, 2003, p. 10.

[17] H. L. Van Trees, Optimum array processing: Part IV of detection, estimation, and modulation theory. John Wiley \& Sons, 2004. 